\title{
Experimental rabies virus infection in Artibeus jamaicensis bats with CVS-24 variants
}

\author{
Jessica E Reid and Alan C Jackson \\ Departments of Medicine and Microbiology and Immunology, Queen's University, Kingston, Ontario, Canada
}

\begin{abstract}
An experimental model of rabies was established in the fruit-eating bat species Artibeus jamaicensis. The infections caused by CVS-N2c and CVS-B2c, which are both stable variants of CVS-24, were compared after inoculation of adult bats in the right masseter muscle. CVS-N2c produced neurologic signs of rabies with paresis, ataxia, and inability to fly, while CVS-B2c did not produce neurologic signs. Bats were sacrificed and the distribution of rabies virus antigen was assessed in tissue sections with immunoperoxidase staining. Both viruses spread to the brain stem and bilaterally to the trigeminal ganglia by days 2 to 3 . CVS-N2c had disseminated widely in the central nervous system (CNS) by day 4 and had involved the spinal cord, thalamus, cerebellum, and cerebral cortex. CVS-B2c had infected neurons in the spinal cord on day 5 and in the cerebellum, thalamus, and cerebral cortex on day 6 . Infected pyramidal neurons of the hippocampus were observed on day 5 in CVS-N2c infection, but infected neurons were never noted in the hippocampus in CVS-B2c infection. CVS-N2c infected many more neurons and more prominently involved neuronal processes than CVS-B2c. CVS-N2c spread more efficiently in the CNS than CVS-B2c. Morphologic changes of apoptosis or biochemical evidence of DNA fragmentation were not observed in neurons with either virus after this route of inoculation. The different neurovirulent properties of these CVS variants in this model were not related to their in vivo ability to induce apoptosis. Journal of NeuroVirology (2001) 7, 511-517.
\end{abstract}

Keywords: apoptosis; encephalitis; rabies; pathogenesis

\section{Introduction}

Bats are important rabies vectors and they now are responsible for the majority of human cases of rabies in the United States and Canada (Noah et al, 1998; Jackson, 2000; Varughese, 2000). Our present knowledge of rabies pathogenesis is largely based on studies in experimental animal models, which were most often performed in rodents (Jackson, 1994, 1997). Few experimental studies have been performed in bat models of rabies, and the previous studies did not use modern techniques. We have developed an experimental rabies model in adult Artibeus jamaicensis bats (Ortega and Castro-Arellano, 2001), because a bat host is a more natural host than

Address correspondence to Alan C Jackson, Kingston General Hospital, Connell 725, 76 Stuart Street, Kingston, Ontario, Canada K7L 2V7. E-mail: jacksona@post.queensu.ca

Received 17 January 2001; accepted 22 June 2001. rodents such as mice or rats. Natural rabies is known to occur in this species of fruit-eating bat (Price and Everard, 1977), although it is not known to be an important rabies vector.

RNA virus strains exist as quasispecies because of high mutation rates due to absence of proofreading and repair and postreplicative error correction mechanisms (Domingo et al, 1996). Two stable rabies virus variants, CVS-N2c (N2c) and CVS-B2c (B2c), have been selected in cell culture from the CVS-24 strain of fixed rabies virus, which is a mouse-adapted strain (Morimoto et al, 1998; Morimoto et al, 1999). The rabies virus glycoprotein of $\mathrm{B} 2 \mathrm{c}$ has 10 amino acid substitutions compared with that of N2c (Morimoto et al, 1998). These virus variants have been observed to have different biological properties: B2c is more pathogenic in suckling mice, expresses higher levels $(4 \mathrm{X})$ of rabies virus glycoprotein, transports less rabies virus nucleocapsid protein in neuronal processes, and is a stronger inducer of apoptosis in primary neuron cultures. N2c is more pathogenic 
for adult mice, expresses lower levels of glycoprotein, and induces less apoptosis in primary neuron cultures (Morimoto et al, 1999). The ability of these virus variants to induce apoptosis in vivo has not yet been reported. Hence, the relevance of the observations of apoptosis in primary neuron cultures has not yet been established in animals. In this work, a comparative pathogenesis study was performed in bats with these two variants after a peripheral route of inoculation.

\section{Results}

\section{Clinical disease}

Bats infected with B2c did not demonstrate definite clinical signs of rabies and there was no mortality. N2c-infected bats developed signs of rabies including leg spasticity and uncoordinated wing movements beginning 4 days p.i. This advanced to ataxia, leg paresis, and inability to fly by 7 days p.i. Bats became moribund between 5 and 7 days p.i.

\section{Rabies virus antigen distribution}

On day 2 p.i., rabies virus antigen was identified in neurons in the ipsilateral trigeminal ganglia of bats infected with both viruses (Figure 1A, C), and by day 3 p.i. both variants had spread to the brain stem and bilaterally to the trigeminal ganglia (Figure 1B), indicating centrifugal spread from the infected brain stem. More neurons in trigeminal ganglia became infected at later time points (Figure 1D). By day 4 p.i., N2c had spread to involve the cerebellum. Infection was present in deep cerebellar nuclei and the Purkinje cell layer and in associated dendritic processes in the molecular layer. B2c did not infect the cerebellum until day 5 p.i. (Figure 2). N2c had disseminated widely throughout the central nervous system by day 4 p.i. Infected neurons were found throughout the cerebral cortex, thalamus, caudate/putamen, and spinal cord. Viral antigen had a widespread distribution in the brains of B2c-infected bats 5 days p.i. with many neurons infected; antigen was detected in the cerebral cortex, thalamus, caudate/putamen, and spinal cord. Subsequent cortical and brain stem involvement by $\mathrm{B} 2 \mathrm{c}$ was minimal compared to the
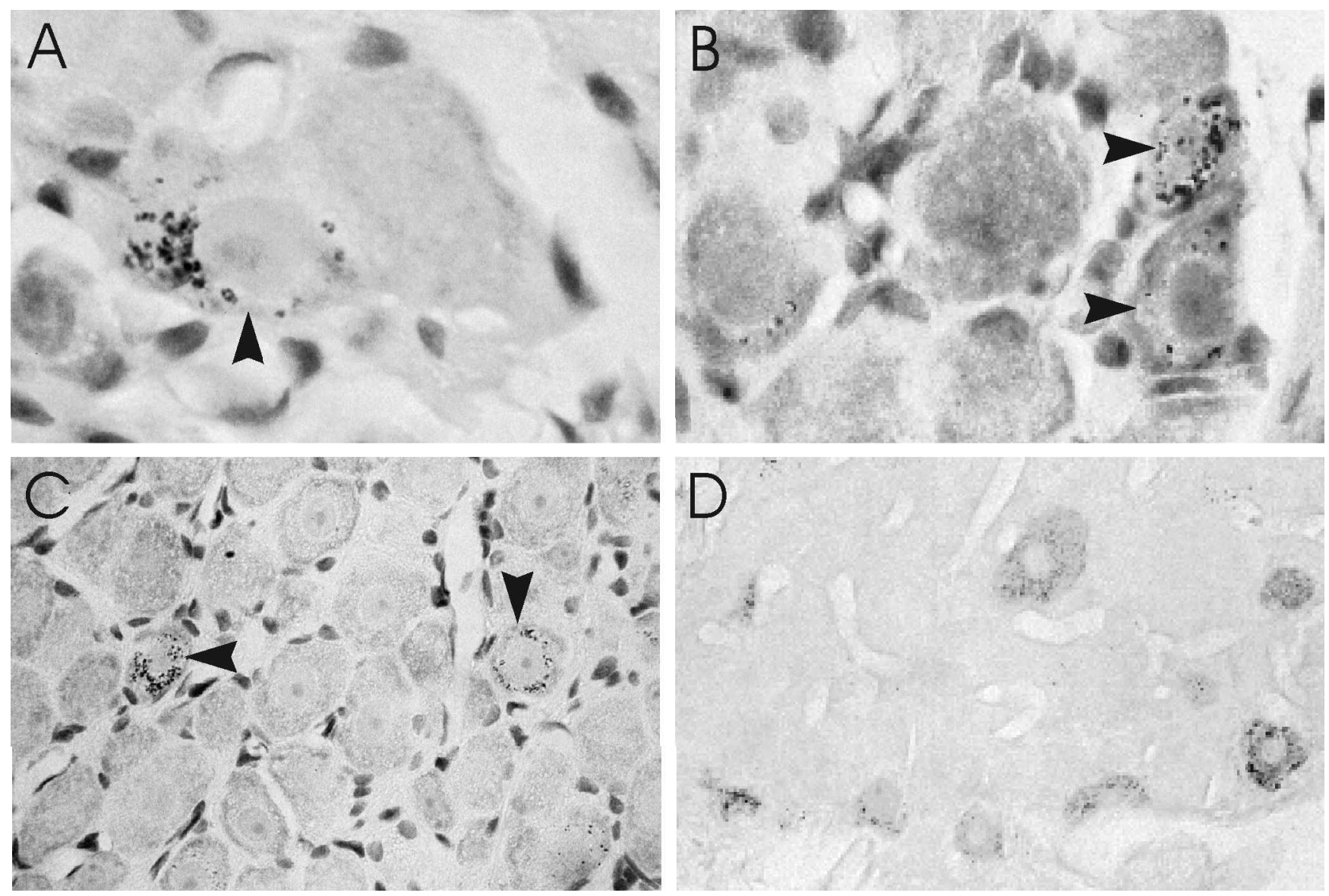

Figure 1 Immunoperoxidase staining for rabies virus antigen in trigeminal ganglia after inoculation of bats with B2c (A, B) and N2c (C, D). Antigen (arrowheads) is seen in neurons in the right (ipsilateral) (A) and left (contralateral) (B) trigeminal ganglia on day 2 p.i. with B2c and in the right trigeminal ganglion on day 2 p.i. with N2c (C). By day 5 p.i., with N2c there is antigen staining in many neurons in the right trigeminal ganglion (D). A, B, C, Immunoperoxidase-hematoxylin; D, Immunoperoxidase. A $\times 1580 ; \mathbf{B} \times 930 ; \mathbf{C} \times 460 ; \mathbf{D} \times 300$. 

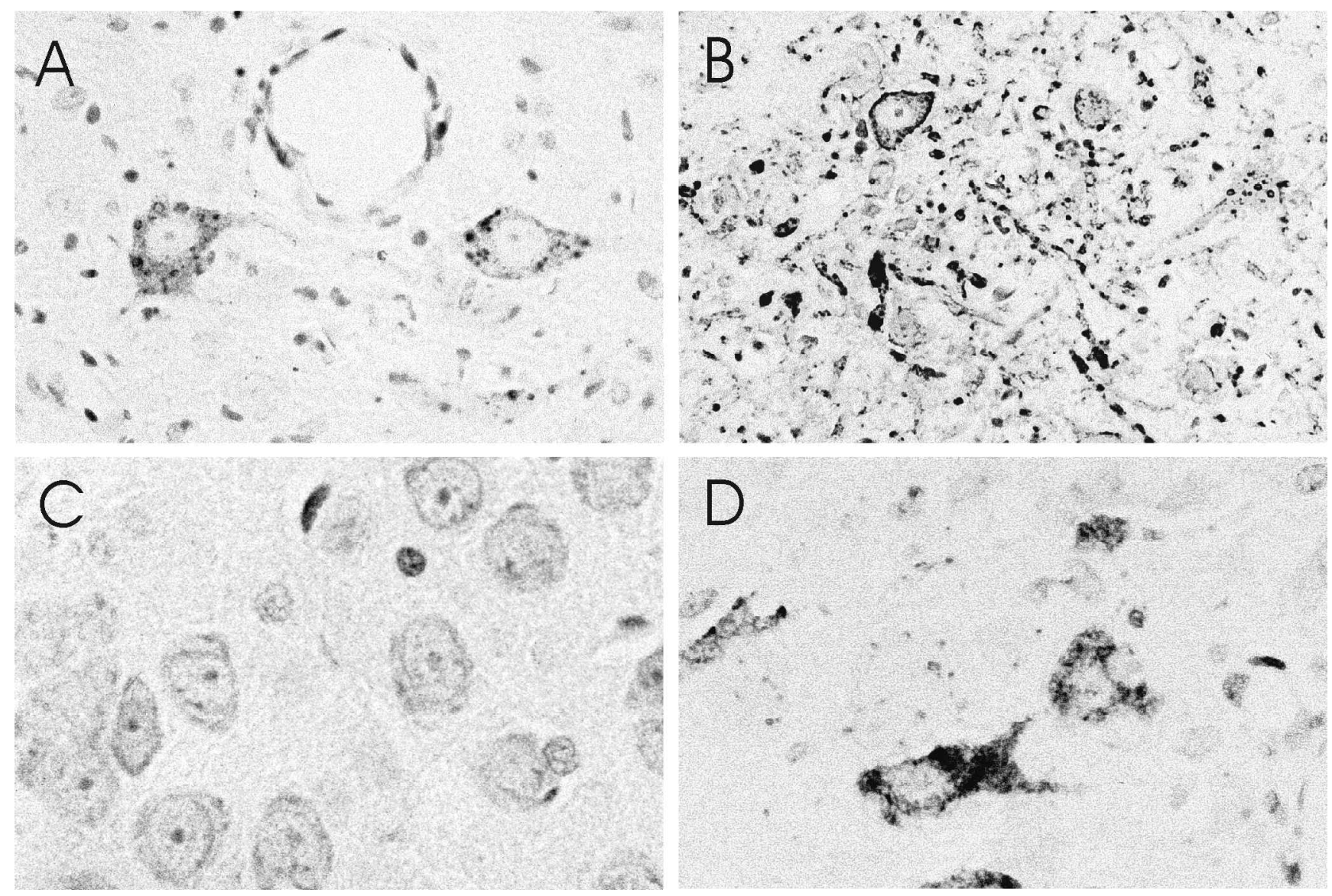

Figure 2 Rabies virus antigen in deep cerebellar nuclei (A, B) and in pyramidal neurons of the hippocampus (CA3 region) (C, D) 6 days after inoculation with B2c (A, C) and N2c (B, D). Prominent infection in neuronal processes are seen after inoculation with N2c (B), but not with B2c (A). Pyramidal neurons of the hippocampus were infected with N2c (D), but not with B2c (C). Immunoperoxidase-hematoxylin. A, B $\times 460 ; \mathbf{C}, \mathbf{D} \times 615$.

infection caused by N2c. The intensity of staining and the number of infected neurons continued to increase up to day 7 p.i. in tissues infected with N2c, whereas staining for viral antigen in bats infected with B2c was maximal at day 6 p.i. as determined by the amount and distribution of immunoreactive signal. The amount of viral antigen in tissues at days 8 and 10 p.i. in bats infected with B2c showed a progressive decline in both signal strength and in the number of neurons infected.

Infection with N2c involved a greater number of neurons at all time points beginning 3 days p.i. and more prominently involved neuronal processes than did B2c (Table 1) as determined by a lesser and more variable amount of staining in brain regions affected by B2c. B2c primarily infected neuronal cell bodies, yet N2c more prominently involved both cell bodies and their associated processes in most brain regions (Figure 2A, B).

N2c had extensively involved brain regions throughout the CNS before infection was observed in the hippocampus. No infected hippocampal neurons were observed at any time point with B2c (Figure 2C), although pyramidal neurons of the hippocampus were initially noted to be infected on day 5 p.i. for N2c (Figure 2D). No infection was noted in the dentate gyrus with either virus.

Table 1 Regional evaluation for presence of rabies virus antigen in neuronal processes as compared to antigen in cell bodies without involvement of processes

\begin{tabular}{lccc}
\hline Brain region & $\begin{array}{c}\text { CVS-B2C } \\
(\mathrm{n}=13)\end{array}$ & $\begin{array}{c}C V S-N 2 C \\
(\mathrm{n}=17)\end{array}$ & Significance \\
\hline Neocortex & 1.00 & 4.00 & $\mathrm{NS}$ \\
Piriform cortex & 1.00 & 5.00 & $\mathrm{NS}$ \\
Caudate/putamen & 1.00 & 4.00 & $P<0.05$ \\
Diencephalon & 1.00 & 5.00 & $P<0.05$ \\
Hippocampus & - & 1.00 & - \\
Cerebellum & 1.00 & 5.00 & $\mathrm{NS}$ \\
Deep cerebellar nuclei & 1.50 & 4.50 & $P<0.05$ \\
Midbrain & 3.00 & 2.00 & $\mathrm{NS}$ \\
Pons/medulla & 1.00 & 5.00 & $P<0.05$ \\
Spinal Cord & 1.00 & 5.00 & $P<0.05$ \\
\hline
\end{tabular}

Rating scale scores expressed as the median score after inoculation with B2c and N2c: 1, 0-20\% of infected cells/field with viral antigen involving processes; $2,21-40 \% ; 3,41-60 \% ; 4,61-80 \%$; $5,81-100 \%$. Significance was assessed by using Fisher's exact test (Statistical Analysis System for Windows, version 8.0, SAS Institute Inc., Cary, NC). 

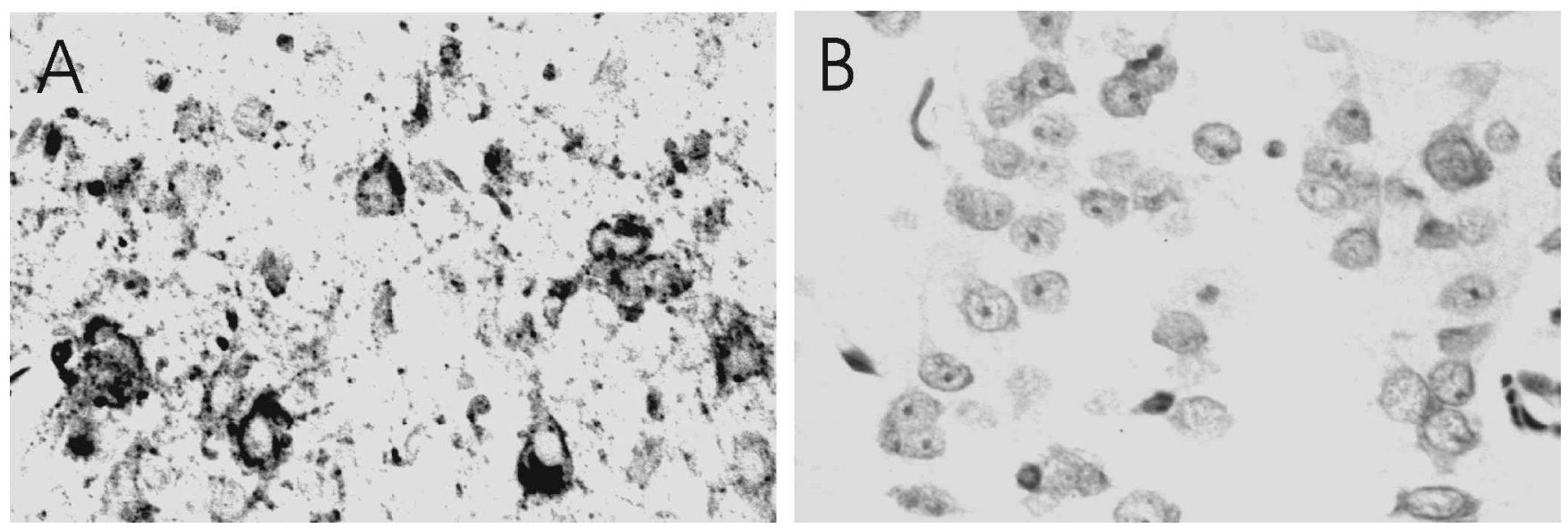

Figure 3 Cerebral cortex (fifth layer) on day 6 p.i. with N2c showing staining for rabies virus antigen (A) and morphologic features (B). Antigen is seen in many cortical neurons and their processes (A). The infected neurons appear morphologically normal without features of apoptosis (B). (A) Immunoperoxidase-hematoxylin; (B) Cresyl violet. A $\times 460$; $\mathbf{B} \times 560$.

\section{Morphologic features}

Typical morphologic changes of apoptosis, including condensation of nuclear chromatin and shrinkage of cellular cytoplasm, were not observed in CNS neurons in either N2c- or B2c-infected bats. Infected neurons throughout the CNS appeared morphologically normal (Figure 3). However, a few small cells compatible with inflammatory cells were noted with condensed nuclei in B2c-infected bats between days 6 and 10 p.i. in the brain stem, diencephalon, caudate/putamen, and cerebral cortex, and they were present in regions with infiltrations of inflammatory cells. Similar cells were also observed on days 6 and 7 in N2c-infected bats in the same regions. More marked leptomeningeal and perivascular inflammatory infiltrates were observed in bats infected with N2c than with B2c, whereas inflammatory infiltrates in the parenchyma were similar with both viruses in all regions (Table 2).

Table 2 Inflammatory cell infiltrate in regional areas of the brain as seen on days $4-6$ postinoculation

\begin{tabular}{lccc}
\hline Brain region & $\begin{array}{c}\text { CVS-B2C } \\
(\mathrm{n}=8)\end{array}$ & $\begin{array}{c}\text { CVS-N2C } \\
(\mathrm{n}=10)\end{array}$ & Significance \\
\hline Leptomeninges & 0.50 & 3.50 & $P<0.05$ \\
Perivascular & 1.00 & 2.00 & $P<0.05$ \\
Neocortex & 0.00 & 0.50 & NS \\
Piriform cortex & 0.00 & 1.00 & NS \\
Diencephalon & 0.00 & 2.00 & NS \\
Cerebellum & 0.00 & 1.00 & NS \\
Midbrain & 0.00 & 0.00 & NS \\
Pons/medulla & 2.25 & 3.00 & NS \\
Spinal cord & 1.00 & 2.00 & NS \\
\hline
\end{tabular}

Rating scale scores expressed as median score after inoculation with B2c and N2c: 0, 0-5 inflammatory cells/high power microscopic field; 1, 6-10 inflammatory cells/field; 2, 11-15 inflammatory cells/field; 3, 16-20 inflammatory cells/field; 4, >20 inflammatory cells/field. Significance assessed by using Fisher's exact test (Statistical Analysis System for Windows, version 8.0).

\section{TUNEL staining}

Scattered TUNEL-positive cells were observed in B2c-infected bats in the brain stem, diencephalon, caudate/putamen, and cerebral cortex on days 6 to 10 p.i., which corresponded to areas that were infiltrated with many inflammatory cells and contained neurons that stained positive for rabies virus antigen. Similar TUNEL-positive cells were observed in the same regions in N2c-infected bats on days 6 and 7 p.i. Positive TUNEL staining was not observed at earlier time points and was not observed in cell types with definite neuronal morphology, including hippocampal neurons. Strong TUNEL staining of neurons was observed on positive control slides pretreated with DNase I.

\section{Discussion}

Two stable variants of CVS-24-B2c and N2c-have been previously characterized, and N2c was found to be more pathogenic in adult mice (Morimoto et al, 1999). We have compared the infections of adult bats after inoculation of these viruses in the masseter muscle. After this route of inoculation, clinical neurologic disease was not noted in the B2c-infected bats. In contrast, N2c-infected bats developed severe and progressive neurologic disease, which was associated with widespread infection of neurons in the CNS.

After inoculation of the viruses in the masseter muscle, there was rapid spread of both viruses to the brain stem and bilateral trigeminal ganglia, indicating centrifugal spread to the contralateral trigeminal ganglia. Hence, differences in the distribution of these viral infections were not yet apparent at these early time points, suggesting that a fundamentally different mechanism of axonal transport within cranial nerves is unlikely for the two viruses. However, 
by day 4, N2c had spread widely in the CNS and had involved the cerebral cortex, thalamus, cerebellum, and spinal cord. B2c had infected spinal cord neurons on day 5 and neurons in the cerebral cortex, thalamus, and cerebellum on day 6. Infection of hippocampal neurons, which occurs late after peripheral routes of inoculation in mouse models (Jackson and Reimer, 1989), was observed on day 5 in N2c infection, but was not noted in B2c infection. In addition, B2c infected a smaller number of neurons than N2c. B2c spread more slowly through the neuroaxis than N2c, which could be explained by less efficient trans-neuronal spread and/or a slower rate of intraneuronal transport, including axonal transport, of B2c than N2c. N2C also induced a more marked inflammatory reaction than $\mathrm{B} 2 \mathrm{c}$ in the leptomeninges and perivascular regions.

The pathways of viral spread of N2C and B2C in this bat model were similar to the pathways for virulent strain CVS-11 and avirulent variant RV194-2 after inoculation into the masseter muscle of adult mice (Jackson, 1991). In both models, the virulent viruses (N2C and CVS-11) infected more neurons than the nonvirulent viruses (B2c and RV194-2). The neuroanatomical pathways result in late infection in the hippocampus in both rabies virus (Jackson and Reimer, 1989) and Borna disease virus (Carbone et al, 1987) infections after peripheral routes of inoculation in rodent models. Relatively inefficient spread may result in the absence of hippocampal infection with the nonvirulent viruses, rather than the hippocampus being a critical site of involvement for neurovirulent infections. A fundamental difference between the findings in the two models was that the avirulent virus (B2c) in the bat model spread through the CNS more slowly than virulent N2c, yet CVS-11 and RV194-2 infected regional areas of the CNS at about the same time (Jackson, 1991). In both of these models, neurovirulence may relate to the burden of infected neurons in the CNS rather than infection of neurons in specific sites.

Neuronal apoptosis occurs in the brains of adult (Jackson and Rossiter, 1997a; Theerasurakarn and Ubol, 1998), suckling (Jackson and Park, 1998), and neonatal (Theerasurakarn and Ubol, 1998) mice infected with CVS-11 by the intracerebral route, and the apoptosis involves more neurons and more neuronal cell types in suckling mice than in adult mice, including both infected neurons (e.g., neurons in the dentate gyrus of the hippocampus) and uninfected neurons (e.g., external granular layer of the cerebellum) (Jackson and Park, 1998). The involvement of uninfected neurons suggests that indirect mechanisms must be important in the induction of apoptosis in vivo in these cells. Street rabies virus strains have also been recently reported to be associated with apoptosis (Ubol and Kasisith, 2000). Apoptosis has also been observed in CNS cells, including spinal cord motor neurons, of mice infected intramuscularly in the hindlimbs with the attenuated
Pasteur strain of rabies virus (Galelli et al, 2000). These mice developed local and irreversible flaccid paralysis. In contrast, nude mice (lacking T cells) did not develop paralysis or significant apoptosis, suggesting that apoptosis in this model is induced by a $\mathrm{T}$ cell-dependent immune response.

Because B2c was observed to cause much more severe apoptosis than N2c in primary neuron cultures (Morimoto et al, 1999), the occurrence and extent of neuronal apoptosis induced by these variants in vivo is of particular interest. Neither of these viruses induced morphologic changes of apoptosis or positive TUNEL staining in neurons after this peripheral route of inoculation, despite infection of many neurons in different regions of the brain. At later time points, probable inflammatory cells showed features of apoptosis based on morphology and TUNEL staining in the infections produced by both viruses. TUNEL staining of infiltrating $\mathrm{T}$ cells and microglia/macrophages undergoing apoptosis has previously been demonstrated in experimental allergic encephalomyelitis (Kohji and Matsumoto, 2000; Ray et al, 2000). Noninfected TUNEL-positive cells were also observed in the vicinity of dying infected neurons in nonfatal paralyzed mice induced by the attenuated Pasteur strain of rabies virus (Galelli et al, 2000).

In mouse models, we have observed much more neuronal apoptosis after the intracerebral and stereotaxic routes of brain inoculation than after peripheral routes of inoculation (A. C. Jackson, unpublished observations). The different neurovirulent properties of the CVS variants in the present model were clearly not related to their in vivo ability to induce apoptosis. In contrast, we have observed neuronal apoptosis in bats inoculated intracerebrally with CVS-11, which was most prominent in neurons in the diencephalon (C. C. Phelan and A. C. Jackson, unpublished observations). Hence, virus-induced apoptosis in primary neuron cultures does not necessarily correlate with the occurrence of apoptosis in vivo. Neuronal apoptosis in viral infections in animal models is complex and it may be influenced by a number of factors, including the age of the host, the viral strain, the route of inoculation, and host defences. Further studies are needed to better understand this phenomenon and also its relevance to natural rabies.

\section{Materials and methods}

\section{Viruses}

Variants N2C and B2c, previously selected from challenge virus standard (CVS)-24 (Morimoto et al, 1998; Morimoto et al, 1999) were obtained from Bernhard Dietzschold (Thomas Jefferson University, Philadelphia, PA). Viral stocks for N2c and B2c were prepared in mouse neuroblastoma cells (NA) for N2C and in BHK-21 cells for B2c. The titer of N2c was $1.3 \times 10^{8}$ 
focus-forming units (FFU)/ml and the titer of B2c was $2.3 \times 10^{8} \mathrm{FFU} / \mathrm{ml}$.

\section{Animals and inoculations}

Adult Artibeus jamaicensis bats (Granby Zoo, Granby, Quebec) were used. Forty bats were inoculated intramuscularly in the right masseter muscle with $4.0 \times 10^{6} \mathrm{FFU}$ of either N2C (7 males and 13 females) or B2c (10 males and 10 females) in a volume of $0.02-0.03 \mathrm{ml}$. Three uninfected control bats ( 2 males and 1 female) were mock-infected intramuscularly in the right masseter muscle with PBS with $2 \%$ fetal bovine serum.

\section{Preparation of tissue sections}

Bats were anaesthetized with methoxyflurane and perfused with buffered $4 \%$ paraformaldehyde. Brains, spinal cords, and trigeminal ganglia of two to four infected bats for each variant were removed daily on days $2-7$ postinoculation (p.i.), and also on days 8 and 10 p.i. for B2c. These samples were fixed by immersion in the same fixative for $18 \mathrm{~h}$ at $4^{\circ} \mathrm{C}$, dehydrated, and paraffin embedded. Coronal sections of brain, transverse sections of brain stem and spinal cord, and sections of trigeminal ganglia were cut at multiple levels on a microtome $(5 \mu \mathrm{m})$. Tissues for histological examination were stained with cresyl violet.

\section{Immunoperoxidase staining}

Tissue sections were stained for rabies virus antigen by the avidin-biotin-peroxidase (ABC) method as previously described (Jackson et al, 1999). Deparaffinized slides were successively reacted with 5\% normal rabbit serum, anti-rabies virus monoclonal antibody 5DF12, which is identical to clone HAM

\section{References}

Carbone KM, Duchala CS, Griffin JW, Kincaid AL, Narayan O (1987). Pathogenesis of Borna disease in rats: evidence that intra-axonal spread is the major route for virus dissemination and the determinant for disease incubation. J Virol 61: 3431-3440.

Domingo E, Escarmis C, Sevilla N, Moya A, Elena SF, Quer J, Novella IS, Holland JJ (1996). Basic concepts in RNA virus evolution. FASEB J 10: 859-864.

Feiden W, Kaiser E, Gerhard L, Dahme E, Gylstorff B, Wandeler A, Ehrensberger F (1988). Immunohistochemical staining of rabies virus antigen with monoclonal and polyclonal antibodies in paraffin tissue sections. Zentralbl Veterinarmed [B] 35: 247-255.

Galelli A, Baloul L, Lafon M (2000). Abortive rabies virus central nervous infection is controlled by T lymphocyte local recruitment and induction of apoptosis. J NeuroVirol 6: 359-372.

Jackson AC (1991). Biological basis of rabies virus neurovirulence in mice: comparative pathogenesis study using the immunoperoxidase technique. J Virol 65: 537540 . with specificity for the rabies virus nucleocapsid protein (Feiden et al, 1988), diluted 1:160 (obtained from Dr. A. I. Wandeler, Animal Diseases Research Institute, Nepean, Ontario), biotinylated rabbit antimouse IgG diluted 1:100 (Zymed Laboratories, San Francisco, CA), 1\% hydrogen peroxide in methanol, avidin-biotinylated horseradish peroxidase complex (Vector Laboratories, Burlingame, CA), 3,3'diaminobenzidine tetrachloride with $0.01 \%$ hydrogen peroxide, and $0.5 \%$ cupric sulfate in $0.15 \mathrm{M}$ sodium chloride. The slides were counterstained with hematoxylin. Tissues from uninfected bats were used as controls and primary antibody was omitted on tissue sections as another control.

\section{DNA nick end labeling of tissue sections \\ (TUNEL staining)}

DNA fragmentation was assessed in situ in sections of infected and control animals using the terminal deoxynucleotidyl transferase-mediated dUTPdigoxigenin nick end labeling (TUNEL) method as previously described, including positive control slides pretreated with DNase I (Jackson and Rossiter, 1997b).

\section{Acknowledgements}

We gratefully thank Cynthia Phelan for technical assistance and Dr. Bernhard Dietzschold (Thomas Jefferson University, Philadelphia, PA) for variants CVS-B2c and CVS-N2c. The advice of Drs. M. Brock Fenton (York University, North York, Ontario) and Michel Delorme (BioDome, Montreal, Quebec) was much appreciated. This work was supported by the Queen's University Violet E. Powell Research Fund.

Jackson AC (1994). Animal models of rabies virus neurovirulence. In: Current Topics in Microbiology and Immunology, Volume 187: Lyssaviruses. Rupprecht CE, Dietzschold B, Koprowski H (eds). Springer-Verlag: Berlin, pp 85-93.

Jackson AC (1997). Rabies. In: Viral Pathogenesis. Nathanson N, Ahmed R, Gonzalez-Scarano F, Griffin DE, Holmes K, Murphy FA, Robinson HL (eds). LippincottRaven: Philadelphia, pp 575-591.

Jackson AC (2000). Rabies. Can J Neurol Sci 27: 278-282.

Jackson AC, Park H (1998). Apoptotic cell death in experimental rabies in suckling mice. Acta Neuropathol 95: 159-164.

Jackson AC, Reimer DL (1989). Pathogenesis of experimental rabies in mice: an immunohistochemical study. Acta Neuropathol 78: 159-165.

Jackson AC, Rossiter JP (1997a). Apoptosis plays an important role in experimental rabies virus infection. $J$ Virol 71: 5603-5607.

Jackson AC, Rossiter JP (1997b). Apoptotic cell death is an important cause of neuronal injury in experimental 
Venezuelan equine encephalitis virus infection of mice. Acta Neuropathol 93: 349-353.

Jackson AC, Ye H, Phelan CC, Ridaura-Sanz C, Zheng Q, Li Z, Wan X, Lopez-Corella E (1999). Extraneural organ involvement in human rabies. Lab Invest 79: 945-951.

Kohji T, Matsumoto Y (2000). Coexpression of Fas/FasL and Bax on brain and infiltrating $\mathrm{T}$ cells in the central nervous system is closely associated with apoptotic cell death during autoimmune encephalomyelitis. J Neuroimmunol 106: 165-171.

Morimoto K, Hooper DC, Carbaugh H, Fu ZF, Koprowski H, Dietzschold B (1998). Rabies virus quasispecies: implications for pathogenesis. Proc Natl Acad Sci USA 95: 3152-3156.

Morimoto K, Hooper DC, Spitsin S, Koprowski H, Dietzschold B (1999). Pathogenicity of different rabies virus variants inversely correlates with apoptosis and rabies virus glycoprotein expression in infected primary neuron cultures. J Virol 73: 510-518.

Noah DL, Drenzek CL, Smith JS, Krebs JW, Orciari L, Shaddock J, Sanderlin D, Whitfield S, Fekadu M, Olson JG, Rupprecht CE, Childs JE (1998). Epidemiology of human rabies in the United States, 1980 to 1996. Ann Intern Med 128: 922-930.

Ortega J, Castro-Arellano I (2001). Artibeus jamaicensis. Mammalian Species 662: 1-9.

Price JL, Everard CO (1977). Rabies virus and antibody in bats in Grenada and Trinidad. J Wildl Dis 13: 131134.

Ray SK, Schaecher KE, Shields DC, Hogan EL, Banik NL (2000). Combined TUNEL and double immunofluorescent labeling for detection of apoptotic mononuclear phagocytes in autoimmune demyelinating disease. Brain Res Brain Res Protoc 5: 305-311.

Theerasurakarn S, Ubol S (1998). Apoptosis induction in brain during the fixed strain of rabies virus infection correlates with onset and severity of illness. J NeuroVirol 4: 407-414.

Ubol S, Kasisith J (2000). Reactivation of Nedd-2, a developmentally down-regulated apoptotic gene, in apoptosis induced by a street strain of rabies virus. J Med Microbiol 49: 1043-1046.

Varughese P (2000). Human rabies in Canada-1924-2000. Can Commun Dis Rep 26: 210-211. 